\title{
Correction
}

\section{Correction: Vegué et al., "On the Structure of Cortical Microcircuits Inferred from Small Sample Sizes"}

In the article "On the Structure of Cortical Microcircuits Inferred from Small Sample Sizes" by Marina Vegué, Rodrigo Perin, and Alex Roxin, which appeared on pages 8498-8510 of the August 30, 2017 issue, an incorrect affiliation for author Alex Roxin was listed. The authors apologize for the oversight. His corrected affiliation is as follows: ${ }^{2}$ Centre de Recerca Matemàtica, Campus de Bellaterra, Edifici C, 08193 Bellaterra, Spain. This correction does not affect the conclusions of the paper. The author line has been corrected on the online PDF version.

DOI:10.1523/JNEUROSCI.2878-17.2017

\section{Correction: Funk et al., "Role of Somatostatin-Positive Cortical Interneurons in the Generation of Sleep Slow Waves"}

In the article "Role of Somatostatin-Positive Cortical Interneurons in the Generation of Sleep Slow Waves" by Chadd M. Funk, Kayla Peelman, Michele Bellesi, William Marshall, Chiara Cirelli, and Giulio Tononi, which appeared on pages 9132-9148 of the September 20, 2017 issue, the authors accidentally left off listing in the References section the Hilscher et al., 2017 citation mentioned in the Discussion section on page 9146. This correction does not affect the conclusions of the paper. The full reference citation has been included on the online PDF version and displayed below.

Hilscher MM, Leão RN, Edwards SJ, Leão KE, and Kullander K (2017) Chrna2-Martinotti cells synchronize layer 5 type A pyramidal cells via rebound excitation. PLOS Biol 15:e2001392.

DOI:10.1523/JNEUROSCI.2902-17.2017 